\title{
Gestion de projet et co-construction : utopie ou voie du succès? Une réflexion exploratoire
}

\author{
Christophe Leyrie ${ }^{a}$, Sonia Boivin ${ }^{b}$
}

RÉSUMÉ. Depuis quelques années, plusieurs initiatives se réclament d'une vision renouvelée des parties prenantes dans les projets, passant d'une gestion « des » parties prenantes à une gestion " pour » les parties prenantes, notamment par la notion de co-construction. Que veut dire co-construire en contexte de gestion et de gestion de projet? Est-il possible d'envisager la co-construction d'un projet industriel ou commercial? Aucune recherche ne semble rendre compte d'une expérience de mise en œuvre de la co-construction comme approche de gestion d'un projet. Cet article propose donc une réflexion exploratoire alimentée par une étude de cas en cours, en documentant les enjeux associés à la structure et au processus décisionnel de projets co-construits. L'implication des parties prenantes et le pouvoir décisionnel qui leur est accordé représentent un changement de paradigme. Malgré les défis que cela suppose, il semble tout de même possible d'envisager de co-construire un projet industriel et commercial.

\begin{abstract}
In recent years, several initiatives have been calling for a renewed vision of stakeholders in projects, moving from management "of" "stakeholder" to management "for" stakeholders, particularly through the notion of co-construction. What does it mean to co-construct in a management and project management context? Is it possible to consider the co-construction of an industrial or commercial project? No research seems to report an experience of implementing co-construction as a management approach to a project. This article therefore proposes an exploratory reflection fed by an ongoing case study, by documenting the issues associated with the structure and decision-making process of co-constructed projects. The involvement of the stakeholders and the decision-making power granted to them represents a paradigm shift. Despite the challenges that this implies, it still seems possible to consider co-constructing an industrial and commercial project.
\end{abstract}

Encore aujourd'hui, bien peu d'auteurs s'accordent sur des définitions précises de critères et de facteurs de succès des projets. Une majorité d'entre eux reconnaissent toutefois l'importance d'une perspective multidimensionnelle et donc d'une réelle prise en compte d'un plus grand nombre et d'une plus grande variété d'acteurs projet dans toute tentative sérieuse d'appréhension de ces concepts centraux (Hughes et al., 2016). Cet élargissement des critères et facteurs de succès traduit ou conditionne en retour une tendance à la transition de visions plutôt utilitaristes des parties prenantes, qui considèrent principalement les intérêts de celles-ci en fonction de leur pouvoir de contribution ou de nuisance, vers des visions plutôt normatives où ces intérêts revêtent une valeur plus intrinsèque (Labelle et Leyrie, 2013). Malheureusement, les appels au passage d'une gestion «des » parties prenantes à une gestion « pour» les parties prenantes ne semblent pas se traduire par la satisfaction d'un plus grand nombre d'acteurs au terme des projets (Eskerod et al., 2015). La vision normative des parties prenantes semble avoir bien du mal à trouver son chemin dans la pratique, faute d'approches pouvant réellement et concrètement permettre une gestion de projet inclusive qui soit à la fois efficace et efficiente.

Depuis quelques années pourtant, on peut relever plusieurs initiatives se réclamant plus ou moins explicitement de cette vision renouvelée des parties prenantes, notamment à travers la notion de co-construction. Au Québec, par

\footnotetext{
${ }^{a}$ Professeur, D.Sc., Université du Québec à Chicoutimi

b Ph. D., psychologue, Adm. A., CIUSS du Saguenay-Lac-Saint-Jean, doctorante et chargée de cours, Université du Québec à Chicoutimi
} 
exemple, la mine Canadian Malartic revendique un engagement clair à cohabiter de façon harmonieuse avec la collectivité grâce à une telle démarche de co-construction. Dans ses communications externes, l'entreprise évoque une "méthode de travail » appuyée sur l'intelligence collective et la créativité et axée sur la recherche de solutions grâce à la collaboration et la recherche de consensus (Canadian Malartic, 2005). Mais si la référence à une telle approche semble effectivement cohérente avec une gestion plus inclusive, peut-on à proprement parler d'une gestion de projet « pour » les parties prenantes qui soit à la fois efficiente et efficace? S'agit-il vraiment d'un exemple de projet co-construit? Est-il même possible d'envisager la co-construction d'un projet industriel ou commercial? Cet article propose une première réflexion exploratoire sur ces questions en tentant de mettre quelques travaux récents sur la co-construction dans la perspective de la pratique de la gestion de projet. Cette réflexion s'appuiera sur l'observation directe d'un projet revendiquant une approche de gestion empreinte de co-construction.

\section{Qu'est-ce que la co-construction?}

Afin de pouvoir considérer la compatibilité de la co-construction avec le pilotage performant d'un projet, il apparaît opportun tout d'abord de tenter de comprendre ce que signifie et ce qu'implique réellement cette approche. Or, malgré son usage de plus en plus en plus répandu, la co-construction ne semble pas bénéficier à ce jour d'une définition stabilisée. Le sens commun nous dicte qu'il s'agit d'une démarche de construction collective qui suppose très probablement la coopération active de plusieurs personnes. Mais que veut concrètement dire cette construction? À quoi correspond son caractère collectif et qu'implique pour les acteurs la coopération attendue d'eux? Quels sont ces acteurs? Selon que l'on interrogera des professionnels ou des chercheurs, et en fonction de leur domaine d'intervention ou de leur appartenance disciplinaire, il est fort probable que l'on obtiendra des réponses sensiblement différentes à ces questions.

Très peu de dictionnaires définissent la coconstruction. Dans un des rares à le faire, le Dictionnaire critique interdisciplinaire de la participation, Akrich (2013) propose la définition suivante : «Ce terme sert à mettre en valeur l'implication d'une pluralité d'acteurs dans l'élaboration et la mise en œuvre d'un projet ou d'une action ». L'auteure évoque un usage diversifié du terme qui est mobilisé dans la presse de façon quasi quotidienne en référence à la coopération entre autorités de différents niveaux dans l'élaboration de politiques territoriales, ou plus généralement dans le cadre de la participation d'acteurs « inhabituels » à diverses initiatives prenant place dans la sphère publique ou la sphère privée. Elle observe une tendance similaire dans la littérature académique où le terme se réfère, avec des nuances, à un processus participatif finalisé, entre autres, en sciences du langage, en psychologie du développement, en sciences de l'éducation, en psychologie ou en science politique. Quoi qu'il en soit, pour Akrich, " il s'agit toujours d'articuler les différentes dimensions des projets en cause et de spécifier des mondes possibles. De ce point de vue, la co-construction suppose des modes d'engagement des acteurs sensiblement plus forts que ceux qui sont associés à la concertation ou à la consultation ».

Tenter de circonscrire la co-construction à partir de travaux de recherche mobilisant la notion conduit à constater l'absence d'une définition semblant pouvoir faire autorité. Ainsi, quand les auteurs ne tiennent pas pour acquis que le sens commun permettra une compréhension partagée de ce qui est entendu par co-construction, ils se voient placés devant la nécessité d'établir la signification que revêt le terme dans leur étude. On pourra alors noter une certaine unanimité dans l'affirmation de la nécessaire distinction à établir entre la coconstruction et des termes tels que participation, implication ou encore coopération, mais on relèvera aussi des nuances dans la façon dont ces distinctions sont articulées et dans le choix des dimensions à souligner dans ce cadre en fonction de la discipline ou du contexte d'usage. Schieb-Bienfait et al. (2014) décrivent, par exemple, la coconstruction d'un projet entrepreneurial comme reposant sur le déploiement de relations réciproques entre partenaires clés lors de la conception du projet d'entreprise. Vaillancourt (2014) revendique quant à lui la création du concept de coconstruction par extension de celui de co-production dans le but d'élargir l'espace conceptuel dans l'examen de la participation du tiers secteur à la construction des politiques publiques. La coconstruction ferait ici référence à la participation non seulement aumoment de leur mise en œuvre 
des politiques publiques, mais aussi au cours de leur conception. Pour Audoux et Gillet (2011), la coconstruction des savoirs se joue dans des relations de mutualité qui permettent d'intégrer et de dépasser la dissymétrie entre soi et l'autre, d'affirmer son identité et de reconnaitre l'altérité. Selon les auteurs, à défaut de cette mutualité, il y a risque d'instrumentalisation d'un partenaire par l'autre, donc de déni de l'altérité, et le degré de mutualité constitue l'un des indicateurs de la co-construction.

Dans un ouvrage récent, Foudriat (2016) tente d'apporter une clarification conceptuelle de la co-construction à partir du double point de vue revendiqué de l'universitaire et de l'intervenant. Il vise ainsi à présenter une réflexion pour définir et conduire des dispositifs co-constructivistes en opposition aux approches trop souvent technicistes et rationnelles adoptées dans les milieux des consultants. S'appuyant sur les similitudes relevées dans les définitions de la co-construction dans différents champs, Foudriat (2016, p. 23) définit celleci comme " un processus volontaire et formalisé par lequel deux ou plusieurs individus (ou acteurs) parviennent à s'accorder sur une définition de la réalité (une représentation, une décision, un projet, un diagnostic) ou une façon de faire (une solution à un problème) ». Il ajoute que « l'accord traduit un compromis sur lequel ces acteurs s'entendent et se reconnaissent ». L'auteur souligne l'ambiguïté de la notion qui peut désigner un résultat, renvoyant au compromis et à la convergence des points de vue, ou bien un processus, renvoyant plutôt à un dispositif spécifique qui peut, ou pas, conduire au résultat. Ce dispositif, caractéristique du processus de co-construction, suppose alors un ensemble de règles, de techniques pouvant être très différent en fonction de l'objet du processus, des catégories d'acteurs parties prenantes, de la nature et du type de délibérations impliquées, des modalités des procédures décisionnelles et de la temporalité fixée. Il s'agit donc d'un processus complexe, éminemment contingent et situationnel, qui se distingue de notions connexes comme la consultation, la concertation ou la coopération par son niveau élevé de délibération et d'implication dans le processus décisionnel.

Dans le but d'alimenter notre réflexion préliminaire, nous retiendrons donc que la notion de co-construction renvoie tout d'abord à un résultat

Revue Organisations \& Territoires • Volume 26 • N 1 1-2 2017 incertain qui repose sur une convergence potentielle plutôt qu'un accord négocié. Cette convergence possible résulte d'un processus volontaire et organisé centré autour d'une délibération entre parties prenantes de la situation objet de la coconstruction.

\section{Que veut dire co-construire en contexte de gestion et de gestion de projet?}

Forts de cette première appréhension très préliminaire de la notion de co-construction, nous pouvons nous interroger sur sa résonance dans le contexte de la gestion, et plus précisément dans celui de la gestion de projet. Dans cette perspective, il est d'emblée possible de repérer un certain nombre de tendances sociétales contemporaines qui renvoient précisément à l'idée de démarche participative et à celle de construction collective, et qui ont eu un impact sur le monde organisationnel à la faveur de logiques de gouvernance et au moyen de la gestion. L'intérêt croissant suscité par le développement durable, l'acceptabilité sociale, la responsabilité sociale des entreprises ou encore la démocratie dans l'entreprise en constituent autant d'exemples. De plus, on notera que la complexification croissante des environnements, désormais qualifiés d'écosystèmes, autant sur les plans stratégique et opérationnel, a contribué aussi à (re)placer l'humain et le social au cœur de la réflexion sur la performance par opposition aux visions dominées par le déterminisme et la rationalisation. L'influence de ces mouvements s'est matérialisée en retour dans la recherche par des tendances managériales elles-mêmes concernées par le collectif et le participatif. Certains plaident ainsi pour un management « réhumanisé » centré sur le collectif, la délibération et une production de valeur équilibrée entre l'entreprise et la société (Saives et al., 2017). D'autres s'inscrivent dans une vision " posthéroïque » du leadership et proposent d'aborder celui-ci avant tout comme un processus de construction collective en opposition aux perspectives individuelles et autoritaires (Crevani, Lindgren et al., 2007). D'autres encore soulignent l'importance de l'intelligence collective et de la coopération dans la prise de décision (Dejours, 2015) ou celle de compétences non plus seulement individuelles, mais aussi collectives, organisationnelles, voire interorganisationnelles (Retour, Picq et al., 2009). Ceci sans oublier l'engouement pour «l'entreprise 
libérée » qualifiée de « dispositif participatif ouvert favorisant une participation couverte » et caractérisée, entre autres, par la participation comme mode de fonctionnement par défaut, la démocratisation de l'innovation, la stimulation de la dynamique entrepreneuriale, la poursuite d'un projet sociétal et le renouvellement du rôle des gestionnaires (Gilbert et al., 2017).

Dans la lignée de ces modèles ou tendances, la co-construction est donc désormais explicitement mobilisée dans des études portant sur la gestion du changement (Autissier et Peretti, 2016), le changement technologique (Bernoux et Gagnon, 2008), les structures de financement des PME (Maazou Elhadji et al., 2015), l'entrepreneuriat (SchiebBienfait et al., 2014), l'implantation de démarche qualité (Perrin et Fabbri, 2016), le leadership (Kean et al., 2011) ou le management stratégique (Phanuel, 2009). Le monde professionnel n'est pas en reste comme en témoigne les recommandations du Conseil patronal de l'environnement du Québec voulant que les projets amorcés par les entreprises soient intégrés harmonieusement dans la communauté en favorisant leur co-construction avec les parties prenantes (CPEQ, 2012) ou encore la fiche pratique émise par la Commission éthique, bonnes pratiques, exemplarité du Mouvement des entreprises de France (MEDEF) qui décrit la coconstruction dans la conduite des affaires comme le transfert par un dirigeant à un groupe de travail du processus de décision dont il a préalablement défini l'objectif et le cadre (Bossis et al., 2017). Cette mobilisation accrue de la notion co-construction dans l'univers de la gestion ne semble pas se traduire pour autant par le développement d'une compréhension pratique commune de ce qu'elle implique réellement, si l'on en juge par le niveau plutôt inégal et limité des descriptions qui sont proposées. Ceci sans parler des cas où le terme est utilisé indistinctement avec la coopération, la participation ou la consultation, ou ceux où il est simplement mentionné sans autre forme d'explication ou de définition.

De façon parallèle, dans le contexte plus spécifique des projets et de leur gestion, plusieurs chercheurs rendent compte de l'existence de divers processus de co-construction avec toutefois, là aussi, un niveau de détails et d'approfondissement assez variable. En 2002, Charue-Duboc et Midler décri- vent, par exemple, une démarche de co-construction dans le cadre de la conception de projets industriels. Leur propos n'est pas de définir la coconstruction, mais plutôt de rendre compte de l'activité d'ingénierie dans le contexte de l'approche concourante du management de projet et plus précisément de la transformation de l'activité de conception. L'ingénieur de procédés doit en effet dans ce cadre passer d'une logique de résolution de problème à une démarche de co-construction de la cible et de la solution. Pour cela, il doit avec l'équipe identifier et faire partager les principales incertitudes, définir des couples hypothèse-solution et enfin piloter la convergence sur les options qui restent ouvertes. Dans la même veine, Hope et Amdahl (2011) évoquent la co-construction dans une étude traitant de la participation des utilisateurs pour un projet de conception de système expert grâce à la mise en œuvre d'une approche agile. Au terme de leur recherche de type ethnographique, les auteurs concluent que la co-construction, qu'ils associent à la participation, l'implication, la collaboration des utilisateurs, ne s'est pas concrétisée aussi facilement que prévu. Des différences professionnelles, des contraintes de coûts et de durée, et des difficultés de traduction entre les pratiques de travail des utilisateurs et les méthodes d'ingénierie des connaissances apparaissent avoir contribué à ces résultats mitigés. Sans définir non plus la co-construction, Olasagasti (2012) propose, à travers une recherche-action dans le cadre d'un projet architectural, que la maitrise d'ouvrage permette une plus grande implication et appropriation des acteurs par la mise en place de dynamiques de coopération et d'échanges. L'accompagnement de cette maitrise doit donc privilégier la transversalité et consister en une intervention en permanente co-construction aux côtés des différents acteurs du projet.

Sous un angle différent, Lindgren et Packendorff (2007) s'intéressent aux processus de construction mutuelle du travail en mode projet et de l'identité professionnelle qu'ils qualifient de co-construction. Sans aborder la signification de ce concept, ils en décrivent les processus constitutifs dans ce cadre : confirmation mutuelle, confirmation/information simultanées et infirmation mutuelle. Neukirch Musca et al. (2014) tentent de comprendre comment se renouvellent ou se redéfinissent les projets dans le cadre de situations ou d'environnements hautement risqués et imprévisibles. À partir d'une étude ethnographique en temps réel et en situation 
impliquant une analyse linguistique de conversations et de narratifs, ils décrivent un processus de co-construction discursive. Ce processus fait de formulation et de reformulation de l'inattendu, de recadrage, de concentration de l'attention et de réaffirmation de la cohésion de l'équipe a permis au groupe de réorienter le projet en en renouvelant collectivement le sens dans une situation particulièrement critique. Toujours en lien avec la construction de sens, Phanuel et Renault (2015) montrent l'utilité d'une démarche de co-construction pour faire émerger une vision partagée d'un projet par ses parties prenantes. À l'occasion d'une recherche qualitative par observation participante, ils mettent ainsi en œuvre une méthode permettant d'identifier et de confronter les souhaitables, non souhaitables, possibles, impossibles et incontournables pour les différents acteurs. Ce processus permet selon les auteurs de co-construire une vision commune du projet, ce qui représente selon eux un enjeu majeur du management de projet.

Sans aucune prétention à une quelconque exhaustivité, ces quelques exemples permettent néanmoins de constater que, tout comme pour la gestion en général, la réflexion sur la gestion de projet en particulier a bien intégré la notion de coconstruction. Les recherches évoquées ici n’offrent toutefois pas de définition claire et uniforme du terme qui est mobilisé dans ces contextes divers et en lien avec des dimensions spécifiques des projets, particulièrement durant leur conception. Aucune d'entre elles ne rend compte d'une expérience de mise en œuvre de la co-construction comme approche de gestion d'un projet en tant que telle et ne peut donc nous informer à ce stade sur la faisabilité et les particularités d'une telle entreprise.

\section{Un exemple de co-construction dans la gestion d'un projet}

Afin de poursuivre et d'alimenter notre réflexion, nous proposerons ici quelques constats préliminaires issus essentiellement d'une étude de cas exploratoire en cours portant sur le processus de co-construction dans la gestion d'un projet de changement organisationnel dans un établissement de santé au Québec. Ce projet de changement s'inscrit dans le contexte d'une fusion de neuf établissements concrétisée par l'adoption d'une loi modifiant l'organisation et la gouvernance du réseau de la santé et des services sociaux adoptée en 2015. La transformation qui en découle est d'une envergure sans précédent au Québec, car cette fusion nécessite une refonte complète du fonctionnement, passant d'une gestion par établissement autonome sur chacun des territoires à une gestion par directions dans l'ensemble de la région. L'une des directions, composée de 200 employés et de 7 cadres intermédiaires, a décidé d'inscrire la gestion de ce projet de changement dans un processus de co-construction afin d'en favoriser l'appropriation. En tentant ainsi de passer d'une gestion « des» parties prenantes à une gestion «pour» les parties prenantes, la propriétaire du projet et le gestionnaire de projet ne pouvaient toutefois s'inspirer de solides assises théoriques ou encore de données probantes. En effet, même si certains ouvrages théoriques ou études mentionnées précédemment peuvent ouvrir la voie, aucun n'est suffisamment spécifique pour fournir à ce stade des lignes directrices claires et éprouvées sur la façon de gérer un projet en coconstruction. L'étude exploratoire menée dans ce contexte tente d'en dessiner un premier contour.

Même s'il ne s'agit pas à proprement dit de présenter ici les résultats de l'étude de cas, ce projet représente une opportunité intéressante dans le cadre de notre réflexion exploratoire. D'une part, il met en œuvre un processus de co-construction comme mode de gestion et, d'autre part, il nous permet un accès direct à ce processus grâce à l'implication de la deuxième auteure dans l'équipe de projet. Les constats préliminaires proposés ici sont donc issus d'une approche longitudinale de nature ethnographique avec une observation participante du projet en temps réel et en situation, ce qui est cohérent avec l'étude d'un processus social tel que la co-construction difficilement observable en extériorité. Une telle approche a permis la collecte et l'analyse de données issues de l'observation et de la participation, mais aussi de carnets de bord et d'entrevues individuelles et de groupes. Même s'il importe de considérer dans nos constats le fait que ce projet ne soit pas de nature industrielle ou commerciale, il est d'emblée possible d'avancer que la gestion d'un projet en co-construction tranche avec les façons de faire traditionnelles et amène plusieurs défis de gestion importants, particulièrement sur les plans de la structure mise en place et du processus décisionnel. 


\section{Constats préliminaires autour de la structure mise en place pour la co-construction}

Gérer un projet en co-construction signifie en adapter le pilotage pour que les parties prenantes y aient une place qui leur permette une réelle remise en cause du projet tout au long du processus (Bernoux et Gagnon, 2008). Cette façon de faire augmente nécessairement la complexité associée au projet, mais devrait en favoriser l'appropriation par les acteurs concernés. Dans le projet dont il est question, les principales parties prenantes que sont les 200 employés et gestionnaires visés par le changement devaient avoir une voix dans l'équipe de projet, mais elles ne pouvaient de toute évidence pas toutes être mises à contribution. Un premier défi pratique était donc associé à la composition de l'équipe sur le plan de sa représentativité et de sa légitimité. Un appel de candidatures a d'abord été effectué avec certaines exigences, dont la stabilité dans le secteur de travail, ainsi que la responsabilité de transmission et de cueillette d'informations avec les équipes de travail dans chacun des secteurs. Parmi les personnes intéressées, un choix a été effectué afin de s'assurer d'une représentativité de mission, de titre d'emploi et de territoire géographique. Une représentante des usagers a de plus été intégrée puisque la transformation associée au projet visait ultimement une amélioration des services pour ces derniers, complétant ainsi une équipe de 20 personnes.

L'équipe étant constituée, le nombre, la fréquence et la durée des rencontres ont été déterminés. Une période de temps, ni trop longue ni trop courte, était jugée nécessaire entre les rencontres pour permettre aux participants une réflexion sur leurs propres points de vue ainsi qu'une ouverture au point de vue des autres. Des rencontres d'une journée toutes les deux semaines ont donc été fixées pour une période de six mois. Il était ensuite essentiel de s'assurer que les acteurs aient un langage commun et une compréhension globale du processus et de l'objet des délibérations afin d'y contribuer de façon optimale. Le processus de coconstruction et la délibération, ses prérequis et ses implications ont donc été expliqués dès la première rencontre. Les connaissances de base jugées nécessaires ont aussi été transmises dans le but de favoriser le compromis et permettre de mieux gérer le risque associé à ce processus dont la résultante n'était pas connue. Ce temps investi a permis une compréhension des réalités de chacun comme le souligne un participant : "Les échanges d'aujourd'hui ont permis une prise de conscience des réalités spécifiques à chacun et du travail quand même exigeant, mais important qu'il y a à faire pour cette harmonisation ».

On peut observer ici dès le départ que la mise en place de la structure temporaire devant mener au processus de co-construction représente certains enjeux intimement liés les uns aux autres. Tout d'abord, l'identification des acteurs à impliquer constitue sans aucun doute un défi important. Comment définit-on les parties prenantes et doiton/peut-on indistinctement toutes les impliquer? Rappelons que la co-construction vise à permettre l'émergence, le développement et l'implantation de nouvelles idées par la confrontation des points de vue et les interactions et qu'elle implique pour les participants un pouvoir de décision réelle. Il est donc important à la fois d'assurer une représentativité adéquate par rapport à l'objet de la démarche, mais aussi de s'assurer d'une composition équilibrée entre les forces en présence. Si une approche inclusive des parties prenantes dans les projets est en effet susceptible de permettre une création de valeur supérieure (Lizarralde et al., 2015), il faut reconnaitre, comme le fait Foudriat (2016), que la définition même des participants peut constituer un objet d'enjeux «pouvant susciter de la part des acteurs dominants, à l'origine de la démarche, des stratégies visant à orienter en leur faveur cette définition en incluant ou excluant certains acteurs » (Foudriat, 2016, p. 27). On doit ainsi notamment composer dans cet exercice avec les procédures et structures hiérarchiques en place dans les organisations et qui peuvent éventuellement avoir une influence à ce stade.

En lien avec la question de la définition des participants au processus se pose aussi la question du moment de cette définition, moment qui renvoie en réalité à l'objet de la co-construction. Dans le cadre du projet à l'étude qui avait pour objectif de redéfinir des façons de faire, l'objet de la coconstruction correspondait en fait à l'objet du projet. La structure de co-construction mise en place correspondait alors logiquement à l'équipe de projet. Mais serait-il nécessaire ou même approprié de conserver la même équipe de 20 personnes pour piloter l'implémentation de ces nouvelles façons de 
faire au jour le jour? Poser cette question c'est déjà en partie y répondre, et l'on peut supposer que dans des contextes d'implémentation il serait justifié de réfléchir à la composition de la structure en gardant à l'esprit l'impératif de diversité des points de vue sur la décision ou le problème objet de la coconstruction. L'équipe responsable du pilotage ne constituera donc pas nécessairement de façon intégrale et équivalente la structure de co-construction qui devra varier en fonction des objets dont elle se saisira. Restera alors à préciser les mécanismes qui détermineront sur quelle base sélectionner ces objets et donc enclencher le processus.

Un autre enjeu lié à la mise en place de la structure de co-construction est représenté par le nombre des participants mobilisés. Il n'existe a priori pas d'indication précise quant à la taille de la structure requise, mais dans la plupart des situations cette approche inclusive va probablement se solder par un nombre accru d'acteurs impliqués. Comme il a pu être vérifié à l'occasion de l'étude de cas, ceci entraîne évidemment certaines difficultés quant à l'animation des rencontres et au processus de délibérations associé, aspects qui seront abordés dans la section suivante portant sur le processus décisionnel. Une telle implication élargie est évidemment nécessaire pour connaitre les exigences, besoins, souhaits et préoccupations en présence, et donc pour gérer " pour » les parties prenantes (Eskerod et al., 2015); mais elle n'est toutefois pas sans conséquence. Dans un contexte d'interdépendance et d'incertitude, l'augmentation du nombre de participants, la variété, la portée et la subtilité de leurs comportements sont déjà depuis un certain temps reconnues comme des sources de complexité importantes dans les projets (Geraldi et Adlbrecht, 2007; Rolstadas et Schiefloe, 2017), ce qui rend notamment nécessaire l'acquisition de compétences spécifiques pour les gestionnaires (Leyrie, 2015). D’un point de vue plus programmatique que stratégique, l'inclusion de nombreuses parties prenantes peut, de plus, entraîner une perte d'attention sur les acteurs envers lesquels la dépendance est la plus grande, c'est-à-dire ceux qui possèdent les ressources les plus critiques pour la réalisation du projet (Eskerod et al., 2016).

On notera finalement que la mise en place de la structure de co-construction implique une préparation des parties prenantes qui doivent connaitre et comprendre ce à quoi ils s'apprêtent à contribuer.
S'ajoutant à la définition des participants, cette étape de préparation et de compréhension préalable peut se révéler chronophage, mais devrait être rendue plus facile à mesure que les situations de co-construction auxquelles les acteurs participent se multiplient. Ce temps de préparation constitue une condition sine qua non du processus de co-construction et en particulier de l'apprentissage coopérant qu'il suppose (Foudriat, 2016).

\section{Constats préliminaires autour du processus décisionnel en co-construction}

Dans un contexte de co-construction comme celui observé, le positionnement de la propriétaire et du gestionnaire de projet diffère de celui qui est normalement attendu d'eux dans un contexte de gestion de projet plus traditionnelle. Le processus de co-construction suppose en effet non seulement que la propriétaire et le gestionnaire de projet s'engagent à participer aux délibérations, mais aussi qu'ils le fassent au même titre que les autres membres de l'équipe du projet. Cela signifie que l'un comme l'autre n'a plus le plein contrôle sur les décisions prises, ce qui constitue un réel changement de paradigme. Comme le mentionne la propriétaire du projet à l'étude, il faut « faire confiance aux gens du terrain. Le produit final ne sera pas le fruit de la direction, mais du groupe de travail ». Cette façon de faire implique donc que la finalité n'est pas connue d'avance, mais construite chemin faisant avec les membres de l'équipe de projet. À cet effet, la propriétaire du projet mentionne qu'« en co-construction, contrairement à d'autres dossiers il faut que j'aie le moins d'idées préconçues pour pouvoir co-construire et que ce ne soit pas du fake ». Pour Foudriat (2016), le processus de co-construction suppose en effet la mise en place d'un espace de délibération, ou espace dialogique, qui puisse garantir des échanges les plus égalitaires possible en réduisant autant que faire se peut les relations de pouvoir : "les modalités d'échanges entre les acteurs parties prenantes doivent ainsi être précisées de façon telle qu'elles rendent difficiles et freinent la reproduction des mécanismes de domination sociale liés aux différences de statut social, de légitimité, d'expertise, de profession, de genre, d'âge, etc. » (Foudriat, 2016, p. 143). Ce partage du pouvoir décisionnel correspond à une approche de la gouvernance qui s'inscrit visiblement dans le 
mouvement de la gestion participative. Le cas examiné montre par ailleurs que cette approche ne se manifeste pas uniquement au niveau du projet, mais aussi au niveau de la direction générale. La nécessaire adhésion aux principes de la délibération au centre de la co-construction par la propriétaire et le gestionnaire de projet témoigne en effet de cette vision partagée et cohérente de la gouvernance. Une telle orientation peut représenter un changement assez radical pour certaines organisations, comme pour celle que nous avons retenue ici, mais elle peut aussi s'avérer un facteur de succès pour les projets. Il apparaît en effet que sous certaines conditions, une organisation privilégiant une gouvernance orientée sur ses parties prenantes plutôt que sur ses seuls actionnaires, y compris dans le cadre de ses projets, serait plus susceptible de réussir ceux-ci (Joslin et Müller, 2016).

En modifiant les lignes d'autorité et de responsabilité, cette situation crée un contexte d'ambivalence à plusieurs égards. Comme nous l'avons observé, les participants doivent ainsi composer avec des attentes contradictoires, et en particulier celle de déterminer l'issue du projet au cours du processus tout en attendant des réponses claires de la part de la direction sur la finalité du projet. Pour le propriétaire d'un projet de changement organisationnel, il s'avère difficile de lâcher prise sur cette finalité qui se dessine chemin faisant, et en même temps rassurer les participants qui, en l'espèce, constituent les personnes visées par le résultat du projet. Ces réflexions de la propriétaire du projet à l'étude viennent illustrer cette difficulté : «En temps normal si on n'était pas en co-construction, ça n'aurait pas de bon sens que je ne sache pas où je m'en vais, ce serait complètement fou. Mais c'est difficile de ne pas savoir où je m'en vais finalement ». "Tu es en co-construction! Si tu as tout décidé d'avance ça va être de la frime! Mais les gens, parce que tu es directrice, ont des attentes, voudraient avoir plus de direction $[\ldots] »$.

De plus, sur la base de ce que nous avons pu constater, les personnes désignées pour participer à la co-construction ne doivent pas simplement rapporter les opinions de leur groupe d'appartenance, mais s'en inspirer pour ensuite s'impliquer dans le processus de délibération, ce qui constitue un défi important pour eux : «[...] les gens, j'ai l'impression, me font confiance. J'espère juste ne pas les décevoir, je leur ai dit ça l'autre jour : Vous savez dans ce genre de processus-là $[. .$.$] c'est donnant-$ donnant $[. .$.$] . Probablement qu'on va être obligés$ de laisser partir quelques affaires. [...] J'espère que je ne vous décevrai pas trop là-dedans ». Les employés n'ont pas réellement conscience de cet impact lorsqu'ils s'impliquent dans l'équipe de projet, même si cela leur est expliqué. Ils en mesurent toute la portée lorsque cela survient concrètement. Cela génère d'ailleurs une certaine pression chez les membres de l'équipe de projet lorsque les délibérations touchent des sujets plus sensibles et que la décision du groupe s'éloigne de celle préconisée par leur équipe d'appartenance. À ce titre, certains participants mentionnent que : «[...] la longue discussion [...] illustre bien la polarisation des points de vue. [...] les gens sont hésitants entre être solidaires de leur groupe ou solidaires de l'équipe. [...] Il y en a qui sont ambivalents. Il y en a pour qui c'est clair ». "J'étais mal à l'aise [...] parce que je me disais que si j'étais dans les mêmes conditions, sûrement que j'aurais le goût de me retirer [...]. Mais en même temps personne ne peut se retirer au moment où on est $[\ldots]$ on doit y aller ensemble ».

Ces différents constats suggèrent selon nous que la mise en ouvre de la co-construction suppose un contexte, une culture particulière caractérisée par des relations de type stewardship. Comme l'explique Davis et al. (1997), en présence d'intérêts concurrents portés par différents actionnaires et parties prenantes, ce type de relations est le fait d'acteurs qui s’identifient fortement à l'organisation et sont hautement dédiés aux valeurs de celles-ci. Pour les auteurs, une philosophie managériale qui repose sur l'engagement et la confiance, et sur une culture basée sur le collectif et la répartition relativement égalitaire du pouvoir, se révélera propice à ce type de relation. Malgré les difficultés parfois représentées par la mise en place d'une telle culture, il a été montré que celle-ci pouvait être particulièrement favorable à la réussite des projets et notamment à la gestion des échéanciers, des budgets et des attentes (Yazici, 2009). D'autre part, le processus de délibération qui met en œuvre les porteurs de différentes visions de la réalité nous apparaît nécessiter une forme de leadership distribué ou partagé qui peut être décrit comme un processus de groupe dans lequel le leadership est distribué parmi les membres de l'équipe et émerge d'eux (Pearce et Sims, 2002). On pourra établir ici un parallèle avec la gestion de projet agile dont l'efficacité repose, entre autres, sur la confiance et le pouvoir donnés 
à des équipes auto-organisées ainsi que sur la communication permanente entre les parties prenantes et l'implication des clients des projets (Fowler et Highsmith, 2001; Shohi et al., 2016). Dans la mouvance agile, l'approche scrum qui se veut une réponse à des problèmes complexes sur la base de la transparence, du contrôle continu et de l'adaptation (Schwaber et Sutherland, 2013) nécessite pour réussir un leader pouvant, entre autres, conduire les autres à se conduire eux-mêmes, partager sa vision et son style de leadership, être un facilitateur transparent et ouvert et maintenir un haut niveau de motivation (Srivastava et Jain, 2016).

Les personnes choisies à titre de membre de l'équipe de projet et par la même occasion comme participant à la co-construction sont donc pleinement impliquées dans le processus décisionnel. Elles ont l'opportunité de prendre des décisions importantes, mais doivent aussi en assumer la responsabilité auprès de leurs pairs. Il est donc essentiel que le mandat soit clairement défini puis accepté par les membres de l'équipe de projet et dans ce sens un mandat leur a ainsi été proposé et a fait l'objet des premières délibérations. Comme le spécifie Bernoux (2010, p. 330), " [1]e projet doit donc être assez précis pour être un véritable projet, mais assez ouvert pour que les acteurs puissent en aménager la partie qui les concernent ». Cette ouverture représente un premier acte concret d'engagement dans le processus de co-construction de la part de la propriétaire du projet, comme en témoigne cette réflexion : "Qu'est-ce que t'es en train de faire, t'es en train de leur demander de définir le mandat! Mais c'est tellement ta job, t'es en train de virer folle sur la co-construction! C'est ça qui se passait dans ma tête. [...] je me disais, ils ont jusque le mandat entre les mains ». C'est aussi un premier engagement posé par les membres de l'équipe de projet, qui comprennent qu'ils en portent également la responsabilité. Comme il s'agit d'un processus itératif, le mandat s'est précisé au cours de l'avancement des travaux. L'équipe a d'ailleurs convenu d'en retirer certains éléments en cours de projet, soit parce que ces derniers n'étaient plus pertinents, soit parce qu'il s'est avéré préférable qu'ils soient portés par les instances hiérarchiques plus directement concernées.

On comprend ici que les différents acteurs doivent composer avec un niveau d'incertitude assez important relativement à l'issue du processus.
Foudriat (2016) mentionne en effet que le compromis qui doit émerger de la délibération demeure une potentialité et que son niveau de qualité ne peut être pris pour acquis : «Du fait de l'inéluctabilité, à la fois des jeux de pouvoir entre acteurs participant à l'espace dialogique et de la liberté relative que conservent ces derniers, il est impossible d'anticiper ni sur le contenu, ni sur le niveau de qualité de l'accord qui sera atteint au terme du processus co-constructiviste » (Foudriat, 2016, p. 147). Pour le gestionnaire responsable et imputable du projet, la situation semble pour le moins inconfortable et difficile à accommoder. On pourra toutefois trouver un éclairage intéressant dans la notion de "situations improvisationnelles ». Pour Vignikin et al. (2016), ce type particulier de situations naît « lorsque des participants sont réunis et doivent accomplir quasi-spontanément (sic) une action collective conduisant à un résultat soumis à un jugement externe ». Prenant appui sur un cadre d'analyse incluant le niveau de pression temporelle, soit l'urgence, et le niveau d'incertitude, soit la nouveauté et l'imprévisibilité, les auteurs recensent et décrivent de telles situations au long de la vie des projets. En particulier, selon cette lecture, des situations de prise de décision en information imparfaite, de structuration d'action collective, de créativité, de gestion de réunion stratégique et participative s'avèrent particulièrement liées à des situations problématiques d'improvisation modérée (Vignikin et al., 2016). Évidemment, une gestion inadaptée de ces situations que l'on peut rapprocher du contexte d'un processus de co-construction peut faire échouer tout le projet. Une des avenues possibles dans ce cas pourrait résider dans l'usage d'un processus de planification fluide par contraste avec l'approche de la planification stratégique conventionnelle. Cette approche reconnait précisément le besoin d'un engagement et d'une participation active des parties prenantes dans la planification stratégique dans le cadre d'un processus de co-construction. Plutôt que développer un plan stratégique de court et long terme avec des buts, des objectifs, un plan d'implémentation et des méthodes de suivi, l'équipe projet travaillera à l'intérieur d'un cadre de travail fluide dans lequel la co-décision, la co-création et l'audit cyclique constitueront des éléments de procédure essentiels (Tiwari et Winters, 2017). 
De façon comparable à la planification stratégique, la planification opérationnelle du projet dans des conditions de co-construction devra être envisagée de façon différente. Le dispositif à mettre en place devra ainsi considérer à la fois des limites temporelles explicites et déterminées rendues nécessaires par le contexte organisationnel et l'incertitude liée au processus délibératif à la temporalité variable et indéterminée (Foudriat, 2016). On pourra encore une fois ici trouver utile de s'inspirer de certaines approches du management de projet agile telle la planification par vagues ou rolling wave planning qui reconnaît la difficulté de commettre rapidement le plan du projet sur la base d'informations incomplètes ou volatiles (Laufer et al., 2015). L'intérêt de cette méthode d'élaboration progressive et itérative est en effet reconnu dans la cadre de projets complexes caractérisés par des phénomènes d'émergence potentiellement similaires à ceux qui marquent les projets en co-construction (Whitney et Daniels, 2013).

La taille du groupe, la durée et la fréquence des rencontres ainsi que les moments de convergence qui ne peuvent être déterminés d'avance amènent ainsi leur lot de défis pour la propriétaire et le gestionnaire de projet qui doivent de plus maintenir la motivation et l'engagement des participants. Comme il a pu être observé, plus le groupe est important plus le processus de délibération peut être long et laborieux, ce qui influence directement en retour la mobilisation des personnes impliquées. En effet, celles-ci ont besoin de voir des résultats tangibles se dessiner. À cet effet, des membres de l'équipe mentionnent qu'" [e]ncore une fois je constate que la grosseur et la diversité du groupe font en sorte que ça multiplie les interventions qui sont faites »; "[o]n avance bien, mais pas au rythme que je voudrais. Je dois patienter sans arrêt ce qui amène un manque de motivation ». En cours de projet, alors que le climat de confiance était établi et que le rythme de travail était installé, des travaux en petits groupes ont été effectués. Cela a permis de mener des délibérations à deux niveaux, d'abord en sous-groupes ensuite en grand groupe. Cela semble avoir favorisé la convergence ou à tout le moins suscité un renouvellement de l'engagement dans le processus de co-construction chez les membres de l'équipe de projet.

\section{Conclusion}

Dans l'objectif de contribuer à matérialiser le passage d'une gestion «des » parties prenantes à une gestion «pour » les parties prenantes dans les projets, la réflexion préliminaire proposée ici avait pour but d'explorer la signification et la faisabilité de co-construire un projet industriel ou commercial. Après avoir constaté l'absence de définition stabilisée et réellement partagée de la co-construction, et en nous appuyant sur les travaux de Foudriat (2016), nous avons retenu qu'il s'agissait à la fois d'un processus volontaire et organisé autour d'une délibération et d'un résultat potentiel exprimant une convergence dans la définition d'une réalité par les participants. Nous avons ensuite pris note de l'intérêt apparent que suscite la coconstruction dans différents domaines et plus particulièrement dans ceux de la gestion et de la gestion de projet comme en témoignent plusieurs initiatives mises de l'avant par des entreprises ou organisations professionnelles et certains écrits scientifiques. Un survol de la littérature n'a cependant pas permis de saisir pleinement les implications et conséquences d'une adoption réelle d'une démarche de co-construction dans la gestion d'un projet.

Afin de poursuivre notre réflexion, nous avons par la suite proposé quelques constats préliminaires issus d'une étude de cas exploratoire en cours portant sur la co-construction d'un projet de changement dans un établissement de santé. Même si ce projet n'est pas de nature industrielle ou commerciale, il nous est apparu pertinent, car il représentait une tentative réelle de co-construire un projet et nous offrait l'opportunité d'y avoir un accès direct, "de l'intérieur », en situation et en temps réel. Cette étude représente donc un exemple de recherche «à travers » un projet qui permet d'approcher son objet non pas comme étant donné, mais plutôt construit, le chercheur étant immergé dans la richesse de l'action et pouvant ainsi rendre compte du projet et de sa gestion (Lalonde et Bourgault, 2013). Les premiers constats ainsi effectués ont montré selon nous le potentiel d'une approche co-constructiviste des projets tout en commençant à en révéler les enjeux et difficultés quant à la gestion. L'implication des parties prenantes en plus grand nombre et le réel pouvoir décisionnel qui leur est accordé au long du projet semblent en effet bien aller dans le sens d'une vraie prise en compte de ces dernières en tant que critères et facteurs de succès 
des projets, et donc d'une gestion « pour » les parties prenantes. Il apparait toutefois aussi qu'une telle approche représente à plusieurs niveaux un véritable changement de paradigme de gestion. Foudriat (2016) résume ce changement en évoquant la nécessité de redéfinir le rapport au temps, à la maitrise et à l'autre.

Un projet en co-construction offre peu de visibilité sur le rythme de progression du processus. Le cadrage traditionnel du projet par étapes distribuées en amont sur le cycle de vie se révèle difficile, bien que l'établissement de limites temporelles demeure possible pourvu qu'elles prennent en compte la nature non déterministe du processus. Le temps supplémentaire nécessaire aux délibérations et à l'émergence des compromis doit être pris en compte sans qu'il soit pour cela possible d'en faire l'estimation selon les méthodes de planification traditionnelles. Selon les parties prenantes et les contextes, cela suppose une compréhension, une adhésion et une participation à la co-construction à différents niveaux de gouvernance dans et hors de l’organisation. Évidemment, cette appréhension différente du temps contribue à modifier le rapport des acteurs à la maitrise du projet co-construit. Un lâcher-prise va devoir se produire à différents niveaux chez différentes parties prenantes. Les approches de planification stratégique et opérationnelle devront s'adapter. L'action planifiée dans un but prédéterminé doit laisser place à une action finalisée à l'intérieur de limites temporelles. Le rapport à l'autre se transforme lui aussi. Le rôle et la place des acteurs évoluent dans un contexte où le projet n'est plus abordé comme une démarche cohérente et organisée rationalisée à partir des seuls points de vue de la direction et des gestionnaires. Ces deniers ne sont plus reconnus comme les seules voix légitimes dans un contexte prenant en compte le formel et l'informel où l'émergence d'une convergence est sujette aux jeux des interrelations et des rapports de pouvoir inhérents à toute action collective. On peut donc percevoir qu'une démarche de co-construction devra prendre place dans une organisation et des projets marqués par une approche de la gouvernance qui repose sur une culture de participation, d'ouverture et de confiance. Dans ce contexte, les acteurs projet, au premier rang desquels les chefs de projet, devront apprendre de nouvelles façons de contribuer aux projets et développer de nouvelles compétences au fur et à mesure des co-constructions.

En réalité, la co-construction dans les projets nous apparaît comme une compétence collective devant être développée au niveau des acteurs, des collectifs, mais aussi des organisations. Dans cette perspective, et pour revenir à notre questionnement initial, il nous semble possible d'envisager de coconstruire un projet industriel ou commercial malgré les défis réels que cela suppose. De toute évidence, il s'agit ici d'une réflexion très exploratoire prenant en compte une revue partielle de la littérature et des données préliminaires portant sur un cas dans un contexte public. Bien que de très nombreuses questions se posent encore à nous, nous croyons toutefois avoir modestement contribué à enrichir la réflexion sur la co-construction en documentant certains des enjeux qu'elle suppose pour la gestion de projet.

\section{RÉFÉRENCES}

Akrich, M. (2003). Co-construction. Dictionnaire critique et interdisciplinaire de la participation. Repéré le 7 juin 2017 à http://www.participation-et-democratie.fr/es/dico/co-construction.

Audoux, C. et Gillet, A. (2011). Recherche partenariale et co-construction de savoirs entre chercheurs et acteurs : l'épreuve de la traduction. Revue Interventions économiques. Repéré le 26 août 2015 à http://interventionseconomiques.revues.org/1347.

Autissier, D. et Peretti, J. M. (dir.) (2016). Les Miscellanées du changement : 2011-2016, les grandes évolutions de la gestion du changement sur 5 ans. Cormelles-le-Royal, France : Éditions EMS.

Bernoux, P. (2010). Sociologie du changement dans les entreprises et les organisations. Paris : Éditions du seuil.

Bernoux, P. et Gagnon, Y. C. (2008). Une nouvelle voie pour réussir les changements technologiques : la co-construction. La Revue des Sciences de Gestion : Direction et Gestion, 5(233), 51-58.

Bossis, G., et al. (2017). Fiche pratique - Pratique de la co-construction (construction collaborative). b. p. Commission éthique, exemplarité - Mouvement des entreprises de France (MEDEF), 44.

Canadian Malartic (2015). Relations avec la communauté : Démarche de co-construction. Repéré le 6 novembre 2017 à http://communaute.canadianmalartic.com/fr/co-construction. 
Charue-Duboc, F. et Midler, C. (2002). L'activité d'ingénierie et le modèle de projet concourant. Concurrent project management and engineering departments. Sociologie du Travail, 44(3), 401-417.

CPEQ (2012). Guide de bonnes pratiques afin de favoriser l'acceptabilité sociale des projets. Montréal, Québec : Conseil patronal de l'environnement du Québec.

Crevani, L., et al. (2007). Shared leadership: a postheroic perspective on leadership as a collective construction. International Journal of Leadership Studies, 3(1), 40-67.

Davis, J. H., et al. (1997). Toward a stewardship theory of management. Academy of Management Review, 22(1), 20-47.

Dejours, C. (2015). Le choix. Souffrir au travail n'est pas une fatalité. Paris, France: Fayard.

Eskerod, P. et Huemann, M. (2014). Managing for stakeholders. Dans J. R. Turner (dir.), Handbook of Project Management (p. 217-232). Aldershot, UK: Gower.

Eskerod, P., et al. (2015). Stakeholder inclusiveness: enriching project management with general stakeholder theory. Project Management Journal, 46(6), 42-53.

Foudriat, M. (2016). La co-construction. Rennes, France : Presses de l’EHESP.

Fowler, M. et Highsmith, J. (2001). The agile manifesto. Repéré le 30 septembre 2017 à http://andrey.hristov.com/fhtstuttgart/The_Agile_Manifesto_SDMagazine.pdf.

Geraldi, J. et Adlbrecht, G. (2007). On faith, fact, and interaction in projects. Project Management Journal, 38(1), 32-43.

Gilbert, P., et al. (2017). L'entreprise libérée, innovation radicale ou simple avatar du management participatif? Gérer et comprendre, 1(127), 38-49.

Hope, K. L. et Amdahl, E. (2011). Configuring designers? Using one agile project management methodology to achieve user participation. New Technology, Work and Employment, 26(1), 54-67.

Hughes, D. L., et al. (2016). An analysis of the components of project success. Success and failure of IS/IT projects (p. 27-43). Cham, Suisse: Springer International Publishing.

Joslin, R. et Muller, R. (2016). The relationship between project governance and project success. International Journal of Project Management, 34(4), 613-626.

Kean, S., et al. (2011). Followers and the co-construction of leadership. Journal of Nursing Management, (19), 507-516.

Labelle, F. et Leyrie, C. (2013). Stakepartner management in projects: A turn-of-the-century turnaround at Alcan. The Journal of Modern Project Management, 1(1), 30-40.

Lalonde, P. L., et Bourgault, M. (2013). Project, project theories, and project research : A new understanding of theory, practice and education for project management. Dans N. Drouin, R. Müller and S. Sankaran (dir.), Novel approaches to organizational project management research (p. 430-451). Copenhagen, Danemark: Copenhagen Business School.

Laufer, A., et al. (2015). What successful project managers do. MIT Sloan Management Review, 56(3), 43-51.

Leyrie, C. (2015). Exploring collective political competence in socially complex projects. The Journal of Modern Project Management, 3(1), 47-57.

Lindgren, M. et Packendorff, J. (2007). Performing arts and the art of performing - On co-construction of project work and professional identities in theatres. International Journal of Project Management, 25(4), 354-364.

Lizarraldre, G., et al. (2015). Stakeholder integration champions and innovation in the built environment. Dans F. Orstavik, A. Dainty et C. Abbott (dir.), Construction Innovation (p. 47-63). Hoboken, NJ: Wiley-Blackwell.

Maazou, E. I., et al. (2015). Entre compétition et coopétition : le rôle stratégique et social des investisseurs dans la co-construction de l'écosystème du financement des PME-ETI au Québec. Vie \& Sciences de l'Entreprise, 1(199), 92-113.

Neukich Musca, G. et al. (2014). "Drop your boat!": The discursive co-construction of project renewal. The case of the Darwin mountaineering expedition in Patagonia. International Journal of Project Management, 32(7), 1157-1169.

Olasagasti, E. (2012). L'assistance à la maîtrise d'ouvrage : quelles modalités d'accompagnement? Le cas d'un projet de reconversion architecturale. Projectics / Proyéctica / Projectique, 2(11-12), 135-146.

Pearce, C. L. et Sims, H. P. (2002). Vertical versus shared leadership as predictors of the effectiveness of change management teams: An examination of aversive, directive, transactional, transformational, and empowering leader behaviors. Group dynamics, 6(2), 172-197.

Perrin, C. et Fabbri, R. (2016). Rôle et posture d'un manager dans la coconstruction de sens commun autour du déploiement d'une démarche qualité dans une organisation de solidarité internationale. Gérer et comprendre, 2(124), 29-40. 
Phanuel, D. (2009). Le management stratégique par les « S.P.I.N.S » : coconstruire un monde partagé. Management \& Avenir, $1(21), 64-79$

Phanuel, D. et Renault, S. (2015). Le partage de vision des acteurs : un enjeu majeur du management de projet. Management \& Avenir, 1(75), 129-145.

Retour, D., et al., (dir.) (2009). Gestion des compétences, nouvelles relations, nouvelles dimensions. Paris, France : Vuibert.

Rolstadås, A. et P. Schiefloe, M. (2017). Modelling project complexity. International Journal of Managing Projects in Business, 10(2), 295-314.

Saives, A. L., et al. (2017). Le management rébumanisé. Montréal, Québec : Chenelières Éducation.

Schieb-Bienfait, N., et al. (2014). Dans quelle mesure le business model (modèle d'affaires) peut-il être un outil d'accompagnement à la co-construction d'un projet entrepreneurial collectif? Humanisme et Entreprise, (316), 27-46.

Schwaber, K. et Sutherland, J. (2017). The scrum guide. Repéré à http://www.scrumguides.org/.

Sohi, A. J., et al. (2016). Does lean \& agile project management help coping with project complexity? Procedia - Social and Behavioral Sciences, (226), 252-259.

Srivastava, P. et Jain, S. (2017). A leadership framework for distributed self-organized scrum teams. Team Performance Management: An International Journal, 23(5/6), 293-314.

Tiwari, R. et Winters, J. (2017). The death of strategic plan: questioning the role of strategic plan in self-initiated projects relying on stakeholder collaboration. International Planning Studies, 22(2), 161-171.

Vaillancourt, Y. (2014). Le tiers secteur dans la co-construction des politiques publiques canadiennes. Canadian Public Policy, 40(Numéro spécial), 4-16.

Vignikin, A., et al. (2016). L'improvisation en tant que situation managériale? Comment évolue-t-elle durant la vie d'un projet? (a) GRH, 1(18), 65-98.

Whitney, K. M. et Daniels, C. B. (2013). The root cause of failure in complex IT projects: complexity itself. Procedia Computer Science, 20, 325-330.

Yazici, H. J. (2009). The role of project management maturity and organizational culture in perceived performance. Project Management Journal, 40(3), 14-33. 